\title{
An Explicit Algorithm for the Construction of 3-Band Wavelet Frames Based on FMRA
}

\author{
Zhaofeng Li ${ }^{1,2, ~ *, ~ Y u a n y u a n ~ Z h a n g ~}{ }^{1,2}$ \\ ${ }^{1}$ College of Science, China Three Gorges University, Yichang, China \\ ${ }^{2}$ Three Gorges Mathematical Research Center, China Three Gorges University, Yichang, China
}

Email address:

Kelly0128@163.com (Zhaofeng Li)

${ }^{*}$ Corresponding author

\section{To cite this article:}

Zhaofeng Li, Yuanyuan Zhang. An Explicit Algorithm for the Construction of 3-Band Wavelet Frames Based on FMRA. Applied and Computational Mathematics. Vol. 7, No. 3, 2018, pp. 155-160. doi: 10.11648/j.acm.20180703.21

Received: April 8, 2018; Accepted: August 4, 2018; Published: August 13, 2018

\begin{abstract}
In 1974, an French engineer, J. Morlet raised the concept of wavelet transform and established the inversion formula through the experience of physical intuition and signal processing and in 1986, the famous mathematician, Y. Meyer, created a real small wave base. From then on, Wavelet transform is a rapidly developing new subfield in mathematics and is used in more and more fields, such as signal analysis, image processing, quantum mechanics and theoretical physics etc. Multiresolution analysis is a systematic method for constructing orthonormal wavelet bases and most of the current dilation is $M=2$ With the development of wavelet transform, $M>2$-band wavelet is known to have advantages over 2-band wavelet in some aspects such as in signal processing. However, there are relatively less results for the case of $M>2$. Based on this fact and inspired by other similar papers, this paper studies the 3-band wavelets and wavelet frames associated with a given refinable function based on frame multiresolution analysis. In this paper, firstly, a sufficient and necessary condition which the refinable function should satisfy for the existence of wavelet frames is showed. Further, an explicit algorithm to construct this frames is worked out and finally, several designed examples are constructed to illustrate this algorithm.
\end{abstract}

Keywords: Frame Multiresolution Analysis, Polyphase Decomposition, Unitary Matrix Extension

\section{Introduction}

In 1974, an French engineer, J. Morlet, who was engaged in oil signal processing, raised the concept of wavelet transform and established the inversion formula through the experience of physical intuition and signal processing, which was not recognized by mathematicians at that time. Fortunately, in 1986, the famous mathematician, Y. Meyer, created a real small wave base.

Compared with Fourier transform and window Fourier transform, wavelet transform is a local transformation of time and frequency, so it can extract information from the signal effectively and carry out multi-scale refinement analysis of function or signal through the operation function of expansion and translation, and solve many difficult problems which can not be solved by Fourier transform, thus the wavelet transform is called the "mathematical microscope".

With the development of wavelet transform, it is used in more and more fields, such as signal analysis, image processing, quantum mechanics and theoretical physics, intelligence of military electronic warfare and weapons, computer classification and recognition, artificial synthesis of music and language etc. It is well known that the multiresolution analysis (MRA for short) is a systematic method for constructing orthonormal wavelet bases [1, 2]. Based on MRA, in 1997 Ron and Shen worked out the unitary extension principle (UEP for short) [3], which is a momentous and convenient tool for the construction of wavelet frames. MRA requires that the translation of refinable function forms an orthonormal or a Riesz base in $V_{0}$ which many refinable functions can't meet. In 1997 Benedetto and Li extended MRA to affine frames and formulated the notion of FMRA which just needs the translation of refinable function forms a frame in $\boldsymbol{V}_{0}$ [4]. Since then many wavelet frames with good properties such as symmetry and compact support in applications have been designed. On the other hand, the interest and effort has revolved around the dilation $M=2$ and there are relatively less results for the case of $M>2$. In fact in multi-rate digital processing, decimation by an $M>2$ ratio has superiorities over $M=2$ and some results have been applied to the scope of engineering [5-11]. Considering the applications and motivated by the work of $[12,13]$, this 
paper studied the 3-band wavelet frames based FMRA in this note.

First introduce the notion of the frame multiresolution analysis (FMRA for short). Fix an integer $M>2$, an FMRA is a family of nested subspaces

$\left\{V_{j}\right\}_{j \in Z}$ of $L^{2}(R)$ such that

1) $\left\{V_{j}\right\} \subset\left\{V_{j+1}\right\}, \quad j \in Z$;

2) $\bigcap_{j \in Z} V_{j}=\{0\}, \quad \overline{\bigcup_{j \in Z} V_{j}}=L^{2}(R)$;

3) $f(x) \in V_{j}$ if and only if $f(M x) \in V_{j+1}$;

4) There exists a function $\varphi(x) \in V_{0}$ such that $\{\varphi(x-n): n \in Z\}$ forms a frame in $V_{0}$.

The function $\varphi(x)$ is called a frame refinable function for the FMRA. In this paper $\varphi(x)$ is assumed to be compactly supported. If replace "a frame" in the above definition by "an orthonormal or a Riesz base", then the standard definition for MRA is obtained.

From the definition of FMRA, it is easy to have

$$
V_{j}=\left\{\sum_{n \in Z} d_{n} \varphi\left(M^{j} x-k\right): \sum_{n \in Z}\left|d_{n}\right|^{2}<\infty\right\}, j \in Z .
$$

Let $W_{j}, j \in Z$ be the orthonormal complement of $V_{j}$ in $V_{j+1}$, then there exists an orthogonal decomposition of $W_{j}$ into $W_{j s}, 1 \leq s \leq M-1$, i.e.

$$
W_{j}=W_{j 1} \oplus W_{j 2} \oplus \cdots \oplus W_{j(M-1)}
$$

and some compactly supported functions $\psi_{s} \in W_{0 s}, 1 \leq s \leq M-1$ such that

$$
W_{j s}=\left\{\sum_{n \in Z} d_{n} \psi_{s}\left(M^{j} x-k\right): \sum_{n \in Z}\left|d_{n}\right|^{2}<\infty\right\}, j \in Z .
$$

Here, if $\left\{\psi_{s}(x-k)\right\}$ is a Riesz base of $W_{0 s}$, $1 \leq s \leq M-1$, then the functions $\psi_{s}, 1 \leq s \leq M-1$ are called the $M$-band wavelets. On the other hand, if a finite family of functions $\Psi=\left\{\psi^{l}, \psi^{2}, \cdots \psi^{N}\right\} \subset V_{1} \quad(N \geq M-1 \quad$ in general) is a frame of $W_{0 s}, 1 \leq s \leq M-1$ and $\Psi$ is called the $M$ - band wavelet frames constructed from FMRA.

FMRA is an important method to construct and character the wavelets. As noted previously, the case of $M>2$ attracted more and more interest and a few relevant literatures appeared in succession [5-11, 14, 15]. However, most of the fruits dealt with the orthonormal wavelets instead of the wavelet frames. For wavelet frames, there are relatively less results [16-18]. The literatures [7, 14, 15] are about orthonormal wavelets with dilation $M>2$. In 2007, Huang and Cheng presented a sufficient condition for an FMRA to form a tight wavelet frame and give an explicit constructing formula of wavelet tight frames by characteristic vectors [14]. Sun et al studied the construction of 3-band wavelet frames with symmetric properties by parameterizations of masks [17]. This paper, firstly gives a sufficient and necessary condition for the refinable function to generate a wavelet frames. And then, the mask matrix is rewrotten in polyphase decomposition and explicitly constructed a wavelet frame using UEP. This method is easily implemented in the computer. The last section is devoted to some examples to illustrate this algorithm.

\section{Preliminaries and Notations}

This sectio will give a complete characteristic of wavelet frames associated with a given refinable function $\varphi(x)$ in terms of their masks.

Let $\varphi(x) \in L^{2}(R)$ with $\hat{\varphi} \in L^{\infty}, \hat{\varphi}$ continuous at 0 , and $\hat{\varphi}(0)=1$ be a frame refinable function. Since $V_{0} \subset V_{1}=$ $\overline{\operatorname{Span}\{\varphi(3 x-n): n \in Z\}}$, there is a sequence $\left\{h_{n}\right\}_{n \in Z} \in l^{2}$ such that

$$
\varphi(x)=\sum_{n \in Z} h_{n} \varphi(3 x-n)
$$

Taking Fourier transform at both sides of (4) leads to

$$
\hat{\varphi}(\omega)=\frac{I}{3} \sum_{n \in Z} h_{n} e^{-\frac{i}{3} \omega n} \hat{\varphi}\left(\frac{\omega}{3}\right) .
$$

Set $H(\omega)=\frac{1}{3} \sum_{n \in Z} h_{n} e^{-i \omega n},(5)$ is equivalent to

$$
\hat{\varphi}(\omega)=H\left(\frac{\omega}{3}\right) \hat{\varphi}\left(\frac{\omega}{3}\right) .
$$

The $2 \pi$-periodic function $H(\omega)$ is called the refinement mask. Similarly, consider the family

$\Psi=\left\{\psi^{l}, \psi^{2}, \cdots \psi^{N}\right\} \subset V_{1}$, there exist $N$ sequences $\left\{g_{n}^{l}\right\}_{l=1,2, \cdots N} \in l^{2}$ such that

$$
\psi^{l}(x)=\sum_{n \in Z} g_{n}^{l} \varphi(3 x-n)
$$

and

$$
\hat{\psi}^{l}(\omega)=\frac{1}{3} \sum_{n \in Z} g_{n}^{l} e^{-\frac{i}{3} \omega n} \hat{\varphi}\left(\frac{\omega}{3}\right)
$$

here and throughout, $l=1,2, \cdots, N$.

Thus there are $N$ wavelet masks. $H(\omega)$ and $G^{l}(\omega)$ formulate a $3 \times(N+1)$ mask matrix $M(\omega)$ as

$$
\left(\begin{array}{lllll}
\mathrm{H}(\omega( & \mathrm{G}^{1}(\omega \omega & \mathrm{G}^{2}(\omega \omega & \cdots & \mathrm{G}^{\mathrm{N}}(\omega \omega \\
\mathrm{H}\left(\omega+\frac{2 \pi}{3}\right) & \mathrm{G}^{1}\left(\omega+\frac{2 \pi}{3}\right) & \mathrm{G}^{2}\left(\omega+\frac{2 \pi}{3}\right) & \cdots & \mathrm{G}^{\mathrm{N}}\left(\omega+\frac{2 \pi}{3}\right) \\
\mathrm{H}\left(\omega+\frac{4 \pi}{3}\right) & \mathrm{G}^{1}\left(\omega+\frac{4 \pi}{3}\right) & \mathrm{G}^{2}\left(\omega+\frac{4 \pi}{3}\right) & \cdots & \mathrm{G}^{\mathrm{N}}\left(\omega+\frac{4 \pi}{3}\right)
\end{array}\right)
$$

Daubechies [2, p322] verified when $N=2$, there exists an orthonormal wavelet base if and only if (9) is unitary, that is 
$M(\omega) M^{*}(\omega)=I_{3}$, for a.e. $\omega$, where

$M^{*}(\omega)$ represents the complex conjugate of the transpose of $M(\omega)$. Inspired by this, this paper hopes that the wavelet frames also will satisfy this unitary property. Huang Yongdong et.al proved that the unitary is a sufficient condition for the existence of a wavelet frame [14]. In the next part, this paper will state a sufficient and necessary condition that the refinable function $\varphi(x)$ should meet to constitute a wavelet frame and if this condition is satisfied, there is an explicit algorithm to construct a tight wavelet frame. Here a note should be emphasized that the wavelet frame constructed by this algorithm only need three wavelet functions $\Psi=\left\{\psi^{1}, \psi^{2}, \psi^{3}\right\}$.

\section{Examples}

This section gives a few examples of tight wavelet frames associated with the cardinal B-splines $N_{m}(x), m \geq 2$ defined inducting by

$$
N_{m}(x)=\int_{0}^{1} N_{m-1}(t-x) d x
$$

with $N_{1}(x)$ denoting the characteristic function of the unit interval [0,1] (see [2, p188]). These Examples can be obtained by the Theorem which listed in the next section. It is well known that the cardinal B-splines play an important role in the development of wavelet theory. Fix the dilation factor $M=3$, the mask of $N_{m}(x)$ is

$$
H_{m}(\omega)=\left(\frac{1-e^{-3 i \omega}}{3-3 e^{-i \omega}}\right)^{m}=\left(\frac{1+e^{-i \omega}+e^{-2 i \omega}}{3}\right)^{m} \text {. }
$$

Since

$$
\begin{aligned}
& \left|H^{m}(\omega)\right|^{2}+\left|H^{m}\left(\omega+\frac{2 \pi}{3}\right)\right|^{2}+\left|H^{m}\left(\omega+\frac{4 \pi}{3}\right)\right|^{2} \leq \\
& \left|H^{l}(\omega)\right|^{2}+\left|H^{l}\left(\omega+\frac{2 \pi}{3}\right)\right|^{2}+\left|H^{l}\left(\omega+\frac{4 \pi}{3}\right)\right|^{2}=1
\end{aligned},
$$

by the above theorem, there exists a tight wavelet frame $\Psi=\left\{\psi^{l}, \psi^{2}, \psi^{3}\right\}$ associated with $N_{m}(x)$.

Example 1 (Haar wavelet) When $m=1$, the B-splines is known as Haar wavelet whose mask $H^{l}(\omega)=\frac{1+e^{-i \omega}+e^{-2 i \omega}}{3}$ and the polyphase components are

$$
H_{1}^{1}(\omega)=H_{2}^{1}(\omega)=H_{3}^{1}(\omega)=\frac{\sqrt{3}}{3} .
$$

This is the special case in which $\left|H_{1}^{1}(\omega)\right|^{2}+\left|H_{2}^{l}(\omega)\right|^{2}$ $+\left|H_{3}^{l}(\omega)\right|^{2}=1$ and therefore $H_{4}^{l}(\omega)=0$. In fact, $H^{l}(\omega)$ generates an orthonormal wavelet base. The polyphase de composition matrix is

$$
\left(\begin{array}{cccc}
\frac{\sqrt{3}}{3} & -\frac{\sqrt{3}}{3} & -\frac{\sqrt{3}}{3} & 0 \\
\frac{\sqrt{3}}{3} & \frac{3+\sqrt{3}}{6} & -\frac{3-\sqrt{3}}{6} & 0 \\
\frac{\sqrt{3}}{3} & -\frac{3-\sqrt{3}}{6} & \frac{3+\sqrt{3}}{6} & 0 \\
0 & 0 & 0 & 1
\end{array}\right)
$$

and the wavelet masks are

$$
\left\{\begin{array}{l}
G^{l}(\omega)=-\frac{\sqrt{3}}{3}+e^{-i \omega} \frac{3+\sqrt{3}}{6}-e^{-2 i \omega} \frac{3-\sqrt{3}}{6} \\
G^{2}(\omega)=-\frac{\sqrt{3}}{3}-e^{-i \omega} \frac{3-\sqrt{3}}{6}+e^{-2 i \omega} \frac{3+\sqrt{3}}{6}
\end{array}\right.
$$

Example 2 (Linear B-splines)

When $m=2, H^{2}(\omega)=\frac{1}{9}\left(1+e^{-i \omega}+e^{-2 i \omega}\right)^{2}$ and the polyphase components are

$$
H_{1}^{2}(\omega)=\frac{\sqrt{3}}{9}\left(1+2 e^{-3 i \omega}\right), \quad H_{2}^{2}(\omega)=\frac{\sqrt{3}}{9}\left(2+e^{-3 i \omega}\right)
$$

and

$$
H_{3}^{2}(\omega)=\frac{\sqrt{3}}{3} \text {. }
$$

There exists $H_{4}^{2}(\omega)=\frac{2 \sqrt{3}}{9}\left(1-e^{-3 i \omega}\right)$ such that

$$
\left|H_{1}^{2}(\omega)\right|^{2}+\left|H_{2}^{2}(\omega)\right|^{2}+\left|H_{3}^{2}(\omega)\right|^{2}+\left|H_{4}^{2}(\omega)\right|^{2}=1 .
$$

For simplify, we set $e^{-3 i \omega}=u$, thus we have

$$
\left(\begin{array}{l}
H_{1}^{2}(\omega) \\
H_{2}^{2}(\omega) \\
H_{3}^{2}(\omega) \\
H_{4}^{2}(\omega)
\end{array}\right)=\left(\begin{array}{c}
\frac{2 \sqrt{3}}{9} \\
\frac{\sqrt{3}}{9} \\
0 \\
-\frac{2 \sqrt{3}}{9}
\end{array}\right) u+\left(\begin{array}{c}
\frac{\sqrt{3}}{9} \\
\frac{2 \sqrt{3}}{9} \\
\frac{\sqrt{3}}{3} \\
\frac{2 \sqrt{3}}{9}
\end{array}\right)
$$

Applying the above algorithm, we obtain the unitary matrix extension as

$$
\left(\begin{array}{cccc}
\frac{\sqrt{3}}{9}(1+2 u) & \frac{4 \sqrt{3}-(13+\sqrt{3}) u}{30} & \frac{4 \sqrt{3}-(3-\sqrt{3}) u}{18} & \frac{8 \sqrt{3}+(24-2 \sqrt{3}) u}{45} \\
\frac{\sqrt{3}}{9}(2+u) & \frac{\sqrt{3}+(11-\sqrt{3}) u}{15} & \frac{\sqrt{3}-(3+\sqrt{3}) u}{9} & \frac{4 \sqrt{3}-(6+4 \sqrt{3}) u}{45} \\
\frac{\sqrt{3}}{3} & -\frac{(3+\sqrt{3}) u}{10} & \frac{(3-\sqrt{3}) u}{6} & -\frac{(6+2 \sqrt{3}) u}{15} \\
\frac{2 \sqrt{3}}{9}(1-u) & -\frac{2 \sqrt{3}+(1+\sqrt{3}) u}{15} & -\frac{2 \sqrt{3}+(3+\sqrt{3}) u}{9} & -\frac{8 \sqrt{3}-(21-4 \sqrt{3}) u}{45}
\end{array}\right)
$$

Thus the tight wavelet frame masks are

$$
\left\{\begin{aligned}
& G^{l}(\omega)=\frac{\sqrt{3}}{3}\left(\frac{4 \sqrt{3}-(13+\sqrt{3}) e^{-3 i \omega}}{30}\right.+\frac{\sqrt{3}+(11-\sqrt{3}) e^{-3 i \omega}}{15} e^{-i \omega} \\
&\left.-\frac{(3+\sqrt{3}) e^{-3 i \omega}}{10} e^{-2 i \omega}\right) \\
& G^{2}(\omega)=\frac{\sqrt{3}}{3}\left(\frac{4 \sqrt{3}-(3-\sqrt{3}) e^{-3 i \omega}}{18}+\frac{\sqrt{3}-(3+\sqrt{3}) e^{-3 i \omega}}{9} e^{-i \omega}\right.\left.+\frac{(3-\sqrt{3}) e^{-3 i \omega}}{6} e^{-2 i \omega}\right) \\
& G^{3}(\omega)=\frac{\sqrt{3}}{3}\left(\frac{8 \sqrt{3}+(24-2 \sqrt{3}) e^{-3 i \omega}}{45}+\frac{4 \sqrt{3}-(6+4 \sqrt{3}) e^{-3 i \omega}}{45} e^{-i \omega}\right. \\
&\left.-\frac{(6+2 \sqrt{3}) e^{-3 i \omega}}{15} e^{-2 i \omega}\right)
\end{aligned}\right.
$$


Example 3 In the above example, if choose $t_{1}=0, t_{2}=1, t_{3}=2, t_{4}=0$, then

$$
\begin{aligned}
& \left(z^{t_{1}} H_{1}(\omega), z^{t_{2}} H_{2}(\omega), z^{t_{3}} H_{3}(\omega), z^{t_{4}} H_{4}(\omega)\right)^{*} \\
= & \left(\frac{\sqrt{3}}{9}\left(1+2 z^{3}\right), \frac{\sqrt{3}}{9}\left(2 z+z^{4}\right), \frac{\sqrt{3}}{3} z^{2}, \frac{2 \sqrt{3}}{9}\left(1-z^{3}\right)\right)^{T} .
\end{aligned}
$$

The corresponding unitary matrix extension is

$$
\left(\begin{array}{cccc}
\frac{\sqrt{3}}{9}\left(1+2 z^{3}\right) & \frac{\sqrt{6}}{9}(1-u) & \frac{\sqrt{2}}{3} & \frac{2}{3} \\
\frac{\sqrt{3}}{9}\left(2+z^{3}\right) & \frac{\sqrt{6}}{18}(4 u-1) & \frac{\sqrt{2}}{6} & -\frac{2}{3} \\
\frac{\sqrt{3}}{3} & -\frac{\sqrt{6}}{6} & -\frac{\sqrt{2}}{2} u & 0 \\
\frac{2 \sqrt{3}}{9}\left(1-z^{3}\right) & \frac{\sqrt{6}}{9}(2 u+1) & -\frac{\sqrt{2}}{3} & \frac{1}{3}
\end{array}\right)
$$

and the tight wavelet frame masks are

$$
\left\{\begin{array}{l}
G^{1}(\omega)=\frac{\sqrt{3}}{3}\left(\frac{\sqrt{6}}{9}\left(1-e^{-3 i \omega}\right)+\frac{\sqrt{6}}{18}\left(4 e^{-3 i \omega}-1\right) e^{-i \omega}\right. \\
G^{2}(\omega)=\frac{\sqrt{3}}{3}\left(\frac{\sqrt{2}}{3}+\frac{\sqrt{2}}{6} e^{-i \omega}-\frac{\sqrt{2}}{2} e^{-5 i \omega}\right) \\
G^{3}(\omega)=\frac{\sqrt{3}}{3}\left(\frac{2}{3}-\frac{2}{3} e^{-i \omega}\right)
\end{array}\right.
$$

It is easy can see that $\psi^{3}$ is anti-symmetric.

\section{Conclusion}

This section state that if the refinable function $\varphi(x)$ satisfies some conditions, there exists a tight wavelet

frame $\Psi=\left\{\psi^{l}, \psi^{2}, \cdots, \psi^{N}\right\} \subset V_{1}$ associated with $\varphi(x)$ by the following theorem.

Theorem: Let $\varphi(x) \in L^{2}(R)$ with $\hat{\varphi} \in L^{\infty}, \hat{\varphi}$ continuous at 0 , and $\hat{\varphi}(0)=1$, be a compactly supported refinable function to generate an FMRA. Then there is a tight wavelet frame associated with $\varphi(x)$ if and only if

$$
|H(\omega)|^{2}+\left|H\left(\omega+\frac{2 \pi}{3}\right)\right|^{2}+\left|H\left(\omega+\frac{4 \pi}{3}\right)\right|^{2} \leq 1 \text {, a.e. } \omega \text {. }
$$

Further, if (10) is satisfied, there exists a compactly supported tight wavelet frame $\Psi=\left\{\psi^{1}, \psi^{2}, \psi^{3}\right\} \subset V_{l}$ associated with $\varphi(x)$.

Before proving this theorem, the correlation of the rows of (9) should be annihilated. To do so and for completeness, a description of the polyphase decomposition technique [2, p318] should be included briefly as follows.

Write $H(\omega), G^{l}(\omega), G^{2}(\omega)$ and $G^{3}(\omega)$ in their polyphase forms respectively as

$$
\left\{\begin{array}{c}
H(\omega)=\frac{1}{\sqrt{3}}\left(H_{1}(\omega)+e^{-i \omega} H_{2}(\omega)+e^{-2 i \omega} H_{3}(\omega)\right) \\
G^{j}(\omega)=\frac{1}{\sqrt{3}}\left(G^{j 1}(\omega)+e^{-i \omega} G^{j 2}(\omega)+e^{-2 i \omega} G^{j 3}(\omega)\right)
\end{array}\right.
$$

Here $j=1,2,3$. Let

$$
N(\omega)=\left(\begin{array}{llll}
H_{1}(\omega) & G^{11}(\omega) & G^{21}(\omega) & G^{31}(\omega) \\
H_{2}(\omega) & G^{12}(\omega) & G^{22}(\omega) & G^{32}(\omega) \\
H_{3}(\omega) & G^{13}(\omega) & G^{23}(\omega) & G^{33}(\omega)
\end{array}\right)
$$

we have

$$
M(\omega)=\frac{1}{\sqrt{3}}\left(\begin{array}{ccc}
1 & e^{-i \omega} & e^{-2 i \omega} \\
1 & e^{-i\left(\omega+\frac{2 \pi}{3}\right)} & e^{-2 i\left(\omega+\frac{2 \pi}{3}\right)} \\
1 & e^{-i\left(\omega+\frac{4 \pi}{3}\right)} & e^{-2 i\left(\omega+\frac{4 \pi}{3}\right)}
\end{array}\right) N(\omega)
$$

and $M(\omega) M^{*}(\omega)=I_{3}$ leads to

$$
N(\omega) N^{*}(\omega)=I_{3} \text {, a.e. } \omega .
$$

Note $H_{1}(\omega), H_{2}(\omega), H_{3}(\omega)$ and $G^{j 1}(\omega), G^{j 2}(\omega)$, $G^{j 3}(\omega)$ are all $2 \pi$-periodic.

Proof of Theorem: The proof is a constructive process inspired by [10] and [11].

Let $H_{1}(\omega), H_{2}(\omega)$ and $H_{3}(\omega)$ be the polyphase components of $H(\omega)$.

Since $|H(\omega)|^{2}+\left|H\left(\omega+\frac{2 \pi}{3}\right)\right|^{2}+\left|H\left(\omega+\frac{4 \pi}{3}\right)\right|^{2} \leq 1$ and

$|H(\omega)|^{2}+\left|H\left(\omega+\frac{2 \pi}{3}\right)\right|^{2}+\left|H\left(\omega+\frac{4 \pi}{3}\right)\right|^{2}=\left|H_{1}(\omega)\right|^{2}+$

$\left|H_{2}(\omega)\right|^{2}+\left|H_{3}(\omega)\right|^{2}$, we have

$$
\left|\boldsymbol{H}_{1}(\boldsymbol{\omega})\right|^{2}+\left|\boldsymbol{H}_{2}(\boldsymbol{\omega})\right|^{2}+\left|\boldsymbol{H}_{3}(\boldsymbol{\omega})\right|^{2} \leq 1
$$

By Riesz Lemma [2, Lemma 6.1.3], there exists a polynomial $H_{4}(\omega)$ which satisfies

$$
\left|\boldsymbol{H}_{1}(\boldsymbol{\omega})\right|^{2}+\left|\boldsymbol{H}_{2}(\boldsymbol{\omega})\right|^{2}+\left|\boldsymbol{H}_{3}(\boldsymbol{\omega})\right|^{2}+\left|\boldsymbol{H}_{4}(\boldsymbol{\omega})\right|^{2}=1 .
$$

Next, multiply a diagonal matrix

$$
\operatorname{diag}\left(e^{-i \omega t_{1}}, e^{-i \omega t_{2}}, e^{-i \omega t_{3}}, e^{-i \omega t_{4}}\right)
$$

to the left of

$$
\left(H_{1}(\omega), H_{2}(\omega), H_{3}(\omega), H_{4}(\omega)\right)^{*}
$$

where $t_{1}, t_{2}, t_{3}$ and $t_{4}$ are so chosen that the matrix $\left(e^{-i \omega t_{1}} H_{1}(\omega), e^{-i \omega t_{2}} H_{2}(\omega), e^{-i \omega t_{3}} H_{3}(\omega), e^{-i \omega t_{4}} H_{4}(\omega)\right)^{*}$ can be written as $\sum_{j=0}^{n} \alpha_{j}^{(0)}\left(e^{-i \omega}\right)^{j}$ with the vectors $\alpha_{j}^{(0)} \in R^{3}$ and $\alpha_{0}^{(0)} \neq 0, \alpha_{n}^{(0)} \neq 0$. For convenience, set $e^{-i \omega}=z$, that is 


$$
\left(z^{t_{1}} H_{1}(\omega), z^{t_{2}} H_{2}(\omega), z^{t_{3}} H_{3}(\omega), z^{t_{4}} H_{4}(\omega)\right)^{*}=\sum_{j=0}^{n} \alpha_{j}^{(0)} z^{j}
$$

(16) leads to

$$
\left(\sum_{j=0}^{n} \alpha_{j}^{(0)} z^{j}\right)^{*} \cdot\left(\sum_{j=0}^{n} \alpha_{j}^{(0)} z^{j}\right)=1
$$

which means $\alpha_{0}^{(0)^{T}} \cdot \alpha_{n}^{(0)}=0$. Now apply the unitary matrix extension technique in [3]. Consider the $4 \times 4$

Householder matrix

$$
H_{1}=I_{4}-\frac{2}{\|v\|^{2}} v v^{T}
$$

here $v=\alpha_{n}^{(0)} \pm\left|\alpha_{n}^{(0)}\right| e_{1}$ with the + and-are so chosen that $v \neq 0 . \quad H_{1} \quad$ is orthonormal and $H_{1} \alpha_{n}^{(0)}=\mp\left|\alpha_{n}^{(0)}\right| e_{1}$, so $\left(H_{1} \alpha_{0}^{(0)}\right)^{T}\left(H_{1} \alpha_{n}^{(0)}\right)=\alpha_{0}^{(0)^{T}}\left(H_{1}{ }^{T} H_{1}\right) \alpha_{n}^{(0)}=0 \quad$. This means the first component of $H_{1} \alpha_{0}^{(0)}$ is 0 . Therefore

$$
\begin{aligned}
& \operatorname{diag}\left(z^{-k}, 1,1,1\right) H_{1}\left(z^{t_{1}} H_{1}(\omega), z^{t_{2}} H_{2}(\omega), z^{t_{3}} H_{3}(\omega), z^{t_{4}} H_{4}(\omega)\right)^{*} \\
& =\sum_{j=0}^{n_{1}} \alpha_{j}^{(1)} z^{j}
\end{aligned}
$$

here $k$ is so chosen that (22) is a polynomial vector with the lowest degree. Also, $\alpha_{0}^{(1)}$ and $\alpha_{n_{1}}^{(1)}$ in (22) are not 0 and $\alpha_{0}^{(1) T} \alpha_{n_{l}}^{(1)}=0$. Repeat this procedure at most $n$ times to get a polynomial matrix

$$
H=H_{s} \operatorname{diag}\left(z^{-k_{s-1}}, 1,1,1\right) H_{s-1} \cdots \operatorname{diag}\left(z^{-k}, 1,1,1\right) H_{1}
$$

here, $1 \leq s \leq n+1$. Then

$$
H \cdot\left(z^{t_{1}} H_{1}(\omega), z^{t_{2}} H_{2}(\omega), z^{t_{3}} H_{3}(\omega), z^{t_{4}} H_{4}(\omega)\right)^{*}= \pm e_{1} \text {, }
$$

that is

$$
\begin{aligned}
& \left(H_{1}(\omega), H_{2}(\omega), H_{3}(\omega), H_{4}(\omega)\right)^{*} \\
= & \operatorname{diag}\left(z^{-t_{1}}, z^{-t_{2}}, z^{-t_{3}}, z^{-t_{4}}\right) H^{*} \operatorname{diag}( \pm 1,1,1,1) e_{1},
\end{aligned}
$$

or

$$
\begin{aligned}
& \left(H_{1}(\omega), H_{2}(\omega), H_{3}(\omega), H_{4}(\omega)\right)^{T} \\
= & \operatorname{diag}\left(z^{t_{1}}, z^{t_{2}}, z^{t_{3}}, z^{t_{4}}\right) H^{T} \operatorname{diag}( \pm 1,1,1,1) e_{1},
\end{aligned}
$$

that means $\left(H_{1}(\omega), H_{2}(\omega), H_{3}(\omega), H_{4}(\omega)\right)^{T}$ is the first column of the unitary matrix

$$
\operatorname{diag}\left(z^{t_{1}}, z^{t_{2}}, z^{t_{3}}, z^{t_{4}}\right) H^{T} \operatorname{diag}( \pm 1,1,1,1)
$$

Write

$$
\begin{aligned}
& \operatorname{diag}\left(z^{t_{1}}, z^{t_{2}}, z^{t_{3}}, z^{t_{4}}\right) H^{T} \operatorname{diag}( \pm 1,1,1,1) \\
= & \left(\begin{array}{llll}
H_{1}(\omega) & G^{11}(\omega) & G^{21}(\omega) & G^{31}(\omega) \\
H_{2}(\omega) & G^{12}(\omega) & G^{22}(\omega) & G^{32}(\omega) \\
H_{3}(\omega) & G^{13}(\omega) & G^{23}(\omega) & G^{33}(\omega) \\
H_{4}(\omega) & G^{14}(\omega) & G^{24}(\omega) & G^{34}(\omega)
\end{array}\right) .
\end{aligned}
$$

By the unitary of (16), the corresponding tight wavelet frame masks can be obtained as

$$
\begin{gathered}
G^{j}(\omega)=\frac{1}{\sqrt{3}}\left(G^{j 1}(\omega)+e^{-i \omega} G^{j 2}(\omega)+e^{-2 i \omega} G^{j 3}(\omega)\right) \\
j=1,2,3
\end{gathered}
$$

The proof of Theorem is complete.

Remark 1: Theorem includes the case of orthonormal wavelet base in which the refinable mask $H(\omega)$ satisfies $|H(\omega)|^{2}+\left|H\left(\omega+\frac{2 \pi}{3}\right)\right|^{2}+\left|H\left(\omega+\frac{4 \pi}{3}\right)\right|^{2}=1$. Example 1 explains this special case.

Remark 2: In the above proof, if choose different $t_{1}, t_{2}, t_{3}, t_{4}$ such that (22) can be written in another equation, then may obtain a different frame as Example 3.

\section{References}

[1] S. Mallat, "Multiresolution approximations and wavelet orthonormal bases of L2 (R)," Transactions of the American mathematical society, vol. 315, pp. 69-87.

[2] Daubechies, "Ten lectures on wavelets," CBMF conference series in applied mathematics 61, SIAM, Philadelphia, 1992.

[3] Ron and Z. Shen, "Affine systems in L2 (Rd): the analysis of the analysis operator," Journal of functional analysis, 148 (1997), 408-447.

[4] J. J. Benedetto and S. Li, "The theorem of multiresolution analysis frames and application to filter banks," Appl. Comput. Harmon. Anal., 5 (1998), 398-427.

[5] K. Soman, P. P. Vaidyanathan and T. Q. Nguyen, "Linear phase paraunitary filter banks: theory, factorizations, and applications," IEEE Trans. Signal Processing, 41 (1993), 3480-3496.

[6] P. Steffen, P. N. Heller, R. A. Gopinath and C. S. Burrus, "Theory of regular M-band wavelet bases," IEEE Trans. Signal Processing, 41 (1993), 3497-3510.

[7] C. Chaux and L. Duval, "Image analysis using a dual-tree M-band wavelet transform," IEEE Transactions on Image Processing, 15 (2006), 2397-2412.

[8] M. K. Mihcak, I. Kozintsev, K. Ramchandran and P. Moulin, "Low-complexity image denoising based on statistical modeling of wavelet coefficients," IEEE Signal Processing Letters, 6 (1999), 300-303. 
[9] L. Gan and K. K. Ma, "A simplified lattice factorization for linear-phase perfect reconstruction filter bank," IEEE Signal Processing Letters, 8 (2001), 207-209.

[10] Chao, Zhang, et al. "Optimal scale of crop classification using unmanned aerial vehicle remote sensing imagery based on wavelet packet transform." Transactions of the Chinese Society of Agricultural Engineering (2016).

[11] Shleymovich M. P., M. V. Medvedev, and S. A. Lyasheva. "Object detection in the images in industrial process control systems based on salient points of wavelet transform analysis." International Conference on Industrial Engineering, Applications and Manufacturing IEEE, (2017):1-6.

[12] C. Chui and W. He, "Compactly supported tight frames associated with refinable functions," Appl. Comp. Harmonic Anal., 8 (2000), 293-319.

[13] W. Lawton, S. L. Lee and Z. Shen, "An algorithm for matrix extension and wavelet construction," Math. Comp., 65 (1996), 723-737.
[14] Y. D. Huang and Z. X. Cheng, "Explicit construction of wavelet tight frames with dilation factor $\alpha$," Acta Mathematica Scientia, 2007, 27A (1), 7-18.

[15] J. J. Sun, Y. Huang, S. Y. Sun and L. H. Cui, "Parameterizations of masks for 3-band tight wavelet frames by symmetric extension of polyphase matrix," Applied Mathematics and Computation, 225 (2013), 461-474.

[16] W. Lawton, S. L. Lee and Z. Shen, "An algorithm for matrix extension and wavelet construction," Math. Comp., 65 (1996), 723-737.

[17] Sun Qiyu, "An Algorithm for the construction of symmetric and anti-symmetric $M$ band wavelets," In Wavelet Applications in Signal and Image Processing VIII, Proceedings of SPIE, 4119 (2000).

[18] B. Han, "Symmetric orthonormal scaling functions and wavelets with dilation factor 4," Adv. Comput. Math., 8 (1998), 221-247. 